\title{
Soluble CD83 ameliorates experimental colitis in mice
}

\author{
J Eckhardt ${ }^{1}$, Kreiser $^{1}, M_{\text {Döbbeler }}^{1}$, C Nicolette ${ }^{2}$, MA DeBenedette ${ }^{2}$, IY Tcherepanova ${ }^{2}$, C Ostalecki ${ }^{3}$, \\ AJ Pommer ${ }^{3}$, C Becker ${ }^{4}$, C Günther ${ }^{4}$, E Zinser ${ }^{1}$, TW Mak ${ }^{5}$, A Steinkasserer ${ }^{1}$ and M Lechmann ${ }^{1}$
}

The physiological balance between pro- and anti-inflammatory processes is dysregulated in inflammatory bowel diseases (IBD) as in Crohn's disease and ulcerative colitis. Conventional therapy uses anti-inflammatory and immunosuppressive corticosteroids to treat acute-phase symptoms. However, low remission rate and strong side effects of these therapies are not satisfying. Thus, there is a high medical need for new therapeutic strategies. Soluble CD83, the extracellular domain of the transmembrane CD83 molecule, has been reported to have interesting therapeutic and immunosuppressive properties by suppressing dendritic cell (DC)-mediated T-cell activation and inducing tolerogenic DCs. However, the expression and function of CD83 in IBD is still unknown. Here, we show that CD83 expression is upregulated by different leukocyte populations in a chemical-induced murine colitis model. Furthermore, in this study the potential of SCD83 to modulate colitis using an experimental murine colitis model was investigated. Strikingly, sCD83 ameliorated the clinical disease symptoms, drastically reduced mortality, and strongly decreased inflammatory cytokine expression in mesenteric lymph nodes and colon. The infiltration of macrophages and granulocytes into colonic tissues was vigorously inhibited. Mechanistically, we could show that sCD83-induced expression of indolamine 2,3-dioxygenase is essential for its protective effects.

\section{INTRODUCTION}

The inflammatory bowel diseases (IBD), Crohn's disease and ulcerative colitis, still represent an eminent health problem. The physiological balance of immunostimulatory and immunosuppressive mechanisms of the gastrointestinal (GI) tract is disturbed in these patients. Both develop chronic and relapsing inflammation of the GI tract, greatly reducing the quality of life. In immunocompetent individuals, these pathologies evolve from cytokine-driven inflammation of the gut. ${ }^{1,2}$ Strong activation of antigen-presenting cells and $\mathrm{T}$ cells has been observed in IBD patients. Colonic and intestinal interstitium of Crohn's disease patients contain substantial numbers of $\mathrm{T}$ helper type 1 (Th1) and Th17 cells associated with excessive expression of interferon (IL)-12/IL-23 and interferon- $\gamma$ (IFN$\gamma) / \mathrm{IL}-17$, whereas there is an increased expression of IL-5 and IL-13 in the colon of ulcerative colitis patients. ${ }^{1,2} \mathrm{~T}$ cell-derived cytokines such as IFN- $\gamma$ and tumor necrosis factor- $\alpha$ (TNF- $\alpha$ ) activate tissue macrophages and granulocytes, driving the inflammation by release of pro-inflammatory cytokines, including TNF- $\alpha$, IL-1 $\beta$, IL-6, IL-12, and mediators as nitric oxide. ${ }^{3}$ Next to the role of macrophages and granulocytes, the bacterial microflora of the gut seems to drive IBD. ${ }^{2,4}$ In spite of intensive research, ulcerative colitis is only curable by surgical removal of the colon. There is still no cure for Crohn's disease. Classic medical therapy uses anti-inflammatory and immunosuppressive drugs such as corticosteroids to treat acutephase symptoms. However, this therapy results in remission of only $\sim 60 \%$ of treated patients with strong side effects. Newer drugs, including anti-TNF- $\alpha$ antibodies, target the proinflammatory cytokines directly. However so far, their therapeutic effect alone or in combination with classic immunosuppressive drugs is limited and involves side effects. ${ }^{2,5}$ Therefore, there is a vital need for new and more efficient therapies with fewer side effects.

\footnotetext{
${ }^{1}$ Department of Immune Modulation at the Department of Dermatology, University Hospital Erlangen, Erlangen, Germany. ${ }^{2}$ Argos Therapeutics, Durham, North Carolina, USA. ${ }^{3}$ Department of Dermatology, University Hospital Erlangen, Erlangen, Germany. ${ }^{4}$ Department of Medicine 1, University Hospital Erlangen, Erlangen, Germany and ${ }^{5}$ The Campbell Family Institute for Breast Cancer Research at Princess Margaret Hospital, University Health Network, Toronto, Ontario, Canada. Correspondence: M Lechmann (matthias.lechmann@uk-erlangen.de)
} 
Recently, the soluble CD83 (sCD83) molecule has been shown to be a strong anti-inflammatory and immunosuppressive mediator. $\mathrm{sCD} 83$ consists of the extracellular domain of the membrane-bound CD83 molecule (mCD83). $\mathrm{mCD} 83$ is expressed with costimulatory properties on mature dendritic cells (DCs) but not on immature DCs. ${ }^{6}$ Next to DCs, it has been shown to be also expressed on activated T and B cells. ${ }^{7}$ Hock et al. ${ }^{8}$ identified SCD83 in the sera of healthy individuals and in the supernatant of mature DCs and B cells. Recently, this group reported increased sCD83 levels in sera of patients with hematological malignancies, ${ }^{9}$ and chronic lymphocytic leukemia patients showed a direct connection of SCD83 concentration in sera and treatment-free survival. ${ }^{10}$ This presumes that sCD83 has physiological impact in vivo and may contribute to the immune escape of malignant disorders. In addition, the release of an immunosuppressive sCD83 molecule has been reported after infection of mature DCs with human cytomegalovirus ${ }^{11}$ representing a new viral immune escape mechanism, as it could be demonstrated that recombinant $\mathrm{sCD} 83$ blocks T-cell stimulation ${ }^{12,13}$ and inhibits DC maturation in vitro. ${ }^{12-14}$ In addition, $\mathrm{sCD} 83$ has also been shown to have a highly interesting pharmacological potential in allogeneic murine skin, heart, and kidney transplantation models and in the experimental autoimmune encephalomyelitis model. ${ }^{14-17}$ A clinical impact of sCD83 in IBD was unknown.

In this study, we used a well-established experimental animal model of IBD, the 2,4-dinitrobenzene sulfonic acid (DNBS)induced colitis, to investigate the therapeutic potential of sCD83. Interestingly, treated animals showed less severe clinical symptoms, reduced macrophage and granulocyte infiltration in colonic interstitium, and decreased expression of inflammatory cytokines. With respect to the prevention of organ transplant rejection by $\mathrm{sCD} 83$, it has previously been reported that sCD83-induced indoleamine 2,3-dioxygenase (IDO) expression is absolutely essential. ${ }^{14}$ Mechanistically, we could show in the reported IBD study here that SCD83-induced IDO expression is absolutely vital, as concurrent application of sCD83 with the IDO-specific inhibitor 1-methyl-tryptophan (1-MT) drastically reduced the immunosuppressive effects of sCD83. In summary, here we present the first proof of principle that $\mathrm{SCD} 83$ is a potent immune modulator in DNBS-induced murine colitis. However, additional studies are needed to further investigate its therapeutic potential for IBD patients.

\section{RESULTS}

\section{CD83 is expressed in the GI tract and MLNs}

CD83 has previously been reported to possess immunomodulatory properties. ${ }^{14-17}$ To investigate the role of CD83 in the gut, we performed multispectral camera analysis of CD83eGFP reporter mice revealing a high CD83 expression in the GI tract of naive mice. Similar as in spleen and peripheral lymph nodes, ${ }^{18}$ we detected strong activity of the CD83 promoter in the cells of mesenteric lymph nodes (MLNs), Peyer's patches, and isolated lymphoid follicles of the intestine (Figure 1a). Monitoring by flow cytometry (Figure 1b) showed that $\sim 30 \%$ of MLN cells and $0.08 \%$ of total colonic cells express $\mathrm{CD} 83$ in naive mice (Figure 1c). The major $\mathrm{CD} 83^{+}$populations in MLNs and colons were B cells (73\%; Figure 1b,d). In MLNs, more $\mathrm{CD} 3{ }^{+} \mathrm{CD}^{+}{ }^{+}(15.5 \%)$ and $\mathrm{CD} 3{ }^{+} \mathrm{CD} 8{ }^{+}(5.6 \%) \mathrm{T}$ cells were observed in the $\mathrm{CD} 83^{+}$population (Figure 1d left) when compared with the colon (7\% and 1.6\%, respectively; Figure 1d, right). However, when myeloid subpopulations were analyzed, more $\mathrm{CD}_{11 \mathrm{c}^{+}}$DCs (10.2\%), $\mathrm{Ly} 6 \mathrm{C}^{+} \mathrm{F} 4 / 80^{-}$monocytes/ neutrophils $(7.8 \%)$, and $\mathrm{Ly}_{6 \mathrm{C}} \mathrm{C}^{-} \mathrm{F} 4 / 80^{+}$macrophages of the naive colon (Figure 1d, right) were part of the $\mathrm{CD} 83^{+}$cells than in MLNs $(1.5 \%, 2.8 \%$, and $2.7 \%$ respectively; Figure 1d, left). CD83 is reported to be an activation marker for DC, B-cell, and T-cell subpopulations. Thus, we monitored CD83 expression in the DNBS colitis model at the peak of disease, i.e., on day 3 after DNBS treatment. Using immunohistochemistry analyses, strong infiltrations of CD83-positive cells were observed in the lamina propria (LP) of DNBS-treated animals that overlapped with areas infiltrated with $\mathrm{CD}^{+}$ cells (Figure 2a,b). In addition, flow cytometry showed a significant increase of $\mathrm{CD}_{3} 3^{+} \mathrm{B} 220^{+}$cells (Figure 2c) and $\mathrm{CD} 33^{+} \mathrm{CD} 3{ }^{+} \mathrm{T}$ cells (Figure $2 \mathrm{~d}$ ) in colonic tissues of animals suffering from DNBS colitis. However, using multiepitope ligand cartography (MELC), we could show that most of the infiltrating leukocytes were $\mathrm{CD}_{11 \mathrm{~b}}{ }^{+} \mathrm{Ly}_{6 \mathrm{G}}{ }^{+}$neutrophil granulocytes (Figure 2e, yellow cells) and $\mathrm{CD}_{11 b^{+}} \mathrm{Ly}_{6} \mathrm{G}^{-}$ monocyte/macrophages (Figure 2e, red cells). For a more detailed definition of the LP-infiltrating $\mathrm{CD}_{3} 3^{+}$leukocytes, we isolated mononuclear cells from murine colonic LP of CD83eGFP reporter mice and analyzed the $\mathrm{CD} 83^{+}$cell populations by flow cytometry (Supplementary Figure S1 online). Here we identified $\sim 10 \%$ of LP cells as CD83 ${ }^{+}$ (Supplementary Figure S1B) that correlated well with histology (Figure 2a). The large majority of $\mathrm{CD} 83^{+}$cells were $\mathrm{B} 220^{+} \mathrm{B}$ cells $(70-80 \%)$, followed by $\mathrm{CD} 11 \mathrm{c}^{+}$DCs (11\%), $\mathrm{CD}^{+} \mathrm{T}$ cells (6\%), and $\mathrm{CD}^{+} 1 \mathrm{~b}^{+}$cells $(4 \%)$ (Supplementary Figure S1D, left). Closer examination proved that most $\mathrm{CD}_{3} 3^{+} \mathrm{T}$ cells were $\mathrm{CD}^{+}$(56\%) and only $13 \%$ were $\mathrm{CD} 8^{+} \mathrm{T}$ cells. Surprisingly, a rather high proportion $(\sim 30 \%)$ were DN T cells (Supplementary Figure S1D, right). Almost all $\mathrm{CD}^{+} 3^{+}$DCs were CD8-negative $\mathrm{CD}_{11 \mathrm{c}^{+}}(91 \%), 5 \% \mathrm{pDCs}$, and only $1 \% \mathrm{CD}^{+} \mathrm{CD}^{+11 \mathrm{c}^{+} \mathrm{DCs}}$ (Supplementary Figure S1F, left). Interestingly, over $50 \%$ of $\mathrm{CD}_{3}{ }^{+} \mathrm{CD} 11 \mathrm{~b}^{+}$cells were eosinophils, $\sim 40 \%$ monocyte/ macrophages, and only $2 \% \mathrm{CD} 1 \mathrm{~b}^{+} \mathrm{Ly} 6 \mathrm{G}^{\mathrm{hi}} \mathrm{F} 4 / 80 \mathrm{neg}$ neutrophils (Supplementary Figure S1F, right).

As it has been reported that $\mathrm{CD} 83^{+}$cells are able to release a soluble form of the CD83 molecule, we next analyzed whether such a soluble form could be detected in the colon. Thus, singlecell suspensions of total colonic tissues were prepared, and then cells were incubated in vitro and supernatants were analyzed by enzyme-linked immunosorbent assay (ELISA) in order to detect sCD83 molecules. Interestingly, especially in cells derived from DNBS-treated animals, an increase of sCD83 could be detected (Figure 2f). Thus, during DNBS colitis: (i) $\mathrm{CD} 3^{+}$cells are recruited to inflamed areas of the colon and (ii) a soluble isoform of $\mathrm{CD} 83$ is released. However, the possible 

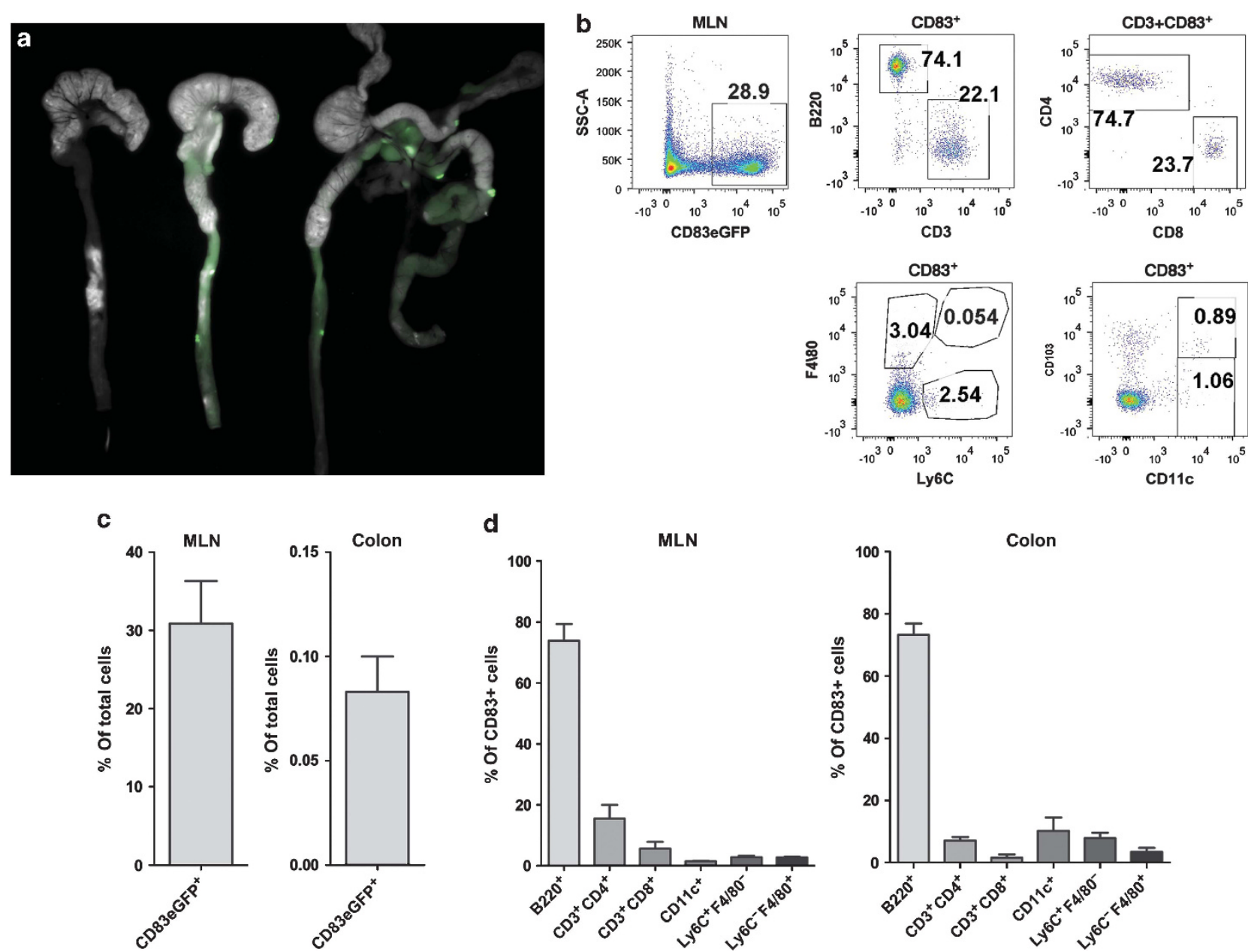

Figure 1 CD83 is highly expressed in gastrointestinal (Gl) tract and mesenteric lymph nodes (MLNs). Freshly extracted GI tissues of (a, left) wild-type or (a, middle, right) CD83eGFP reporter mice were analyzed with a multispectral camera system (Maestro, Cri); CD83 promoter activity (green); (white): autofluorescence of tissue and stool; presented data are spectral unmixed images. (b) Flow cytometric analysis of subpopulations in MLNs gated on $\mathrm{CD} 3 \mathrm{eGFP}^{+}$cells (left) of a naive CD83eGFP reporter mouse. Average distribution of CD83eGFP ${ }^{+}$cells in (c, left) total MLNs and (c, right) colonic tissue together with (d) CD83eGFP ${ }^{+}$subpopulations was monitored by flow cytometry $(n=5)$.

biological significance regarding the presence of sCD83 during colitis was not known and has thus subsequently been addressed.

\section{sCD83 treatment ameliorated symptoms of DNBS-induced colitis}

Previously, it has been reported by our and other groups that soluble CD83 molecules are able to inhibit murine DCmediated T-cell activation in vitro and murine immune responses in the autoimmune experimental autoimmune encephalomyelitis as well as in allogeneic transplantation models in vivo. ${ }^{12-17}$ Because of the interesting expression pattern of CD83 in the gut and the detection of sCD83 in experimental IBD, we investigated the therapeutic effect of sCD83 on inflammatory processes of the GI tract. After we saw upregulation of endogenous sCD83 in the well-established DNBS-induced colitis, we used this model to induce acute inflammation and ulceration in the colons of mice. The clinical features of this model are hemorrhagic diarrhea, significant loss of body weight, and large bowel wall thickening. ${ }^{19}$ The experimental setting was as follows: mice were inoculated with DNBS on day 0 and treated with $\mathrm{sCD} 83$ on days $-1,0$, and +1 with $100 \mu \mathrm{g}$ sCD83 intraperitoneally (Supplementary Figure S2). As expected, DNBS treatment of BALB/c mice resulted in a transient colitis characterized by weight loss, diarrhea, edema, ulceration, and loss of tissue architecture. Clinical disease symptoms, as assessed by macroscopic and histological damage scores, as well as severity of the DNBSinduced colitis, peaked at day 3 after injection and resolved thereafter. The first very striking effect of sCD83 was the extremely diminished mortality of DNBS $+\mathrm{sCD} 83$-treated mice (Figure 3a). Analyses of the weight kinetics also underlined the protective effect of $\mathrm{sCD} 83$ (Figure $3 \mathbf{b}$ ). DNBS + sCD83-treated mice showed a recovery between days 2 and 3 and gained weight on day 3, whereas the DNBS-only-treated animals had an average weight loss of $22 \%$ 


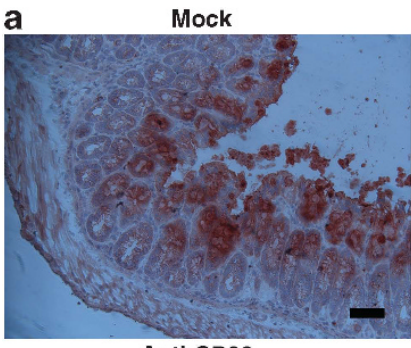

Anti-CD83

b

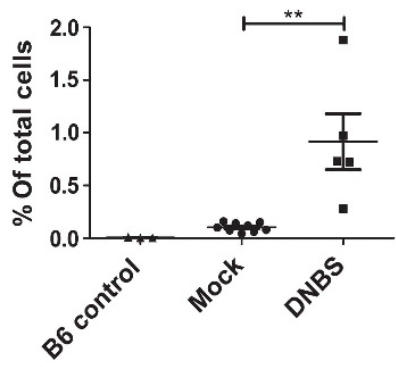

DNBS

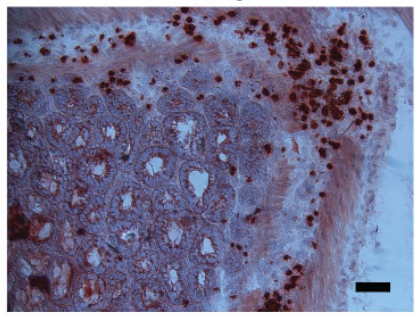

Anti-CD83

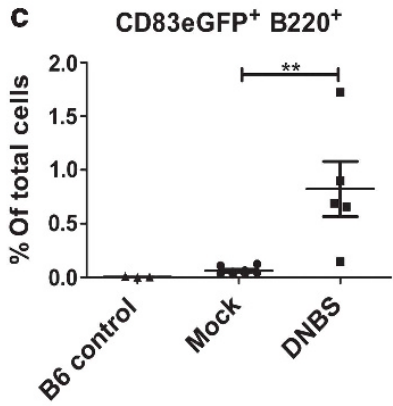

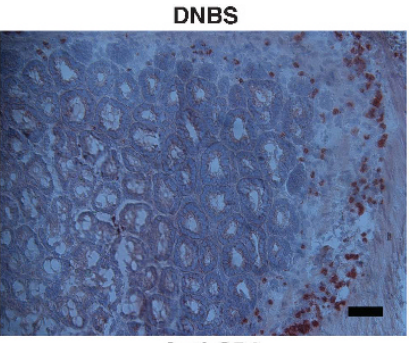

Anti-CD8

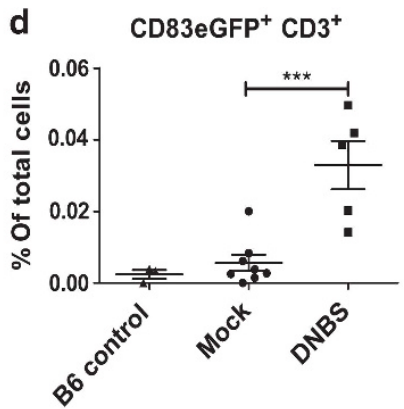

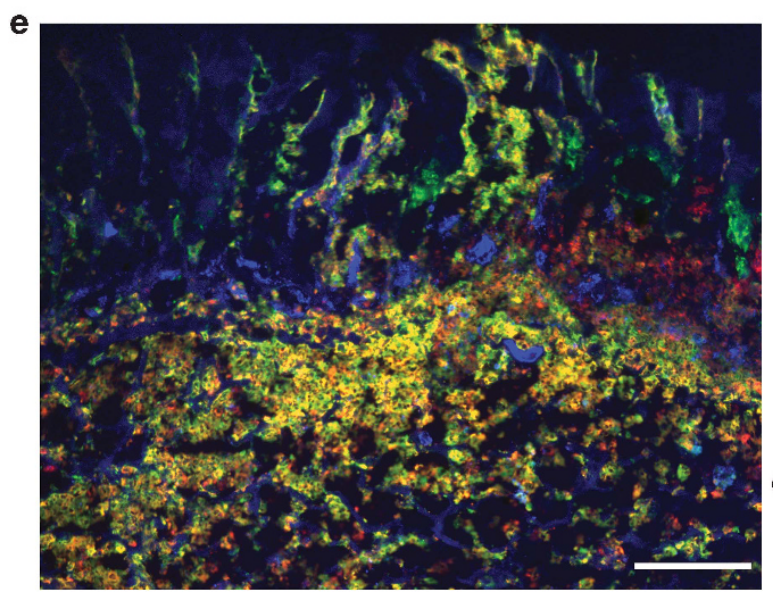

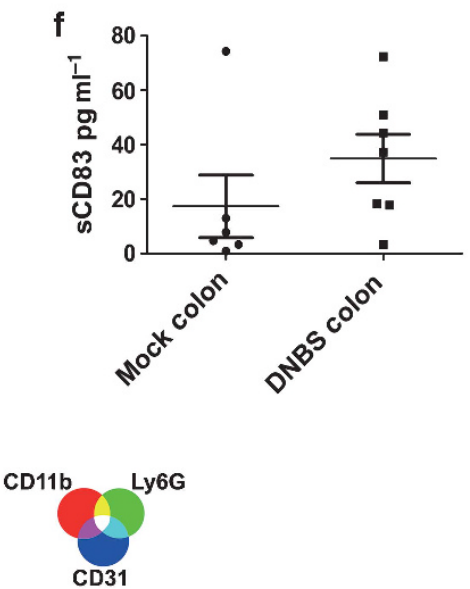

Figure 2 CD83 expression is upregulated in colon during experimental colitis. (a) Immunostaining for CD83 and CD8 in colonic frozen sections of untreated Balb/c mice (mock) and 2,4-dinitrobenzene sulfonic acid (DNBS)/ethanol (4 mg)-treated animals $72 \mathrm{~h}$ after treatment. DNBS colitis animals show lamina propria (LP) infiltration of $\mathrm{CD}_{3} 3^{+}$cells (middle) at similar sights as infiltrating CD8 ${ }^{+}$cells (right); microphotographs were taken at $\times 20$ magnification; bars $=50 \mu \mathrm{m}$. (b-d) Flow cytometry of untreated (mock) and DNBS/ethanol (4 mg)-treated CD83eGFP reporter compared with $\mathrm{C} 57 \mathrm{BI} / 6$ control confirmed increased infiltration of $\mathrm{CD} 3^{+}$cells into colonic tissues $72 \mathrm{~h}$ after treatment (b) with infiltrating $\mathrm{CD}^{+} 3^{+} \mathrm{B}^{220}{ }^{+}$cells (c) and $\mathrm{CD}_{3} 3^{+} \mathrm{CD}^{+}$T cells (d); ( $\left.n=3-9\right)$. (e) RGB image of immunofluorescence microscopy (multiepitope ligand cartography (MELC)) of colonic tissue day 3 after DNBS treatment showed infiltrating CD11b $^{+}$(red) and CD11b ${ }^{+}$Ly6G $^{+}$cells (yellow). CD11b: red, Ly6G: green and CD31: blue; bar $=10 \mu \mathrm{m}$. (f) Soluble CD83 (sCD83) enzyme-linked immunosorbent assay (ELISA) showed release of sCD83 by colonic single-cell suspensions of mock and DNBS mice (day 3) incubated for $5 \mathrm{~h}(n=6-7) .{ }^{* \star} P<0.01,{ }^{* \star *} P<0.001$, analysis of variance (ANOVA) Tukey's multiple comparison test.

at the peak of disease on day 3. At the end, DNBS + sCD83 mice showed an average weight loss of only $13 \%$ on day 3 . Further comparison of the macroscopic scores on day 3 indicated a strongly increased pathology in DNBS-only mice (Figure 3c,d). Histological analysis of hematoxylin and eosin tissue stainings confirmed these findings (Figure $\mathbf{3 e}$ ). There were decreased infiltrating leukocytes, less severe destruction of the colonic architecture, and a minor loss of goblet cells observed in sCD83-treated animals (Figure 3e,f). Thus, sCD83 treatment revealed a highly potential protective effect during DNBS colitis.

\section{sCD83 treatment inhibited the infiltration of inflammatory leukocytes into the colon}

DNBS induces $\mathrm{T}$ cell-mediated inflammation within the colonic mucosa that is characterized by infiltration of activated $\mathrm{T}$ cells, macrophages, and neutrophils into the colon wall. ${ }^{20}$ T cell-derived cytokines as IL- 2 , TNF- $\alpha$, and IFN- $\gamma$ drive gut inflammation by activating macrophages, monocytes, and granulocytes to release large amounts of TNF- $\alpha$, IL-1 $\beta$, IL-6, IL-12, and reactive oxide species. Especially, the neutrophils and monocytes are the main infiltrating injury-driving phagocytic leukocytes. $^{3}$ 
a

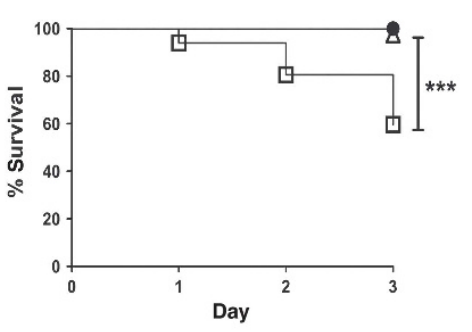

C

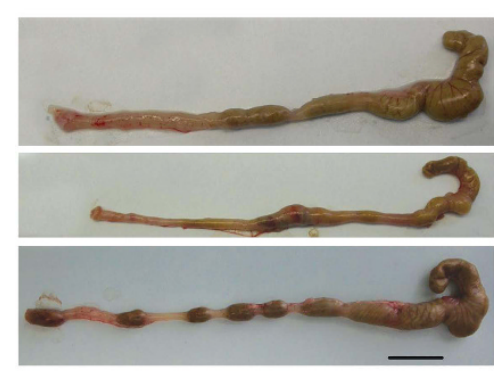

b - Ethanol

$\triangle D N B S+s C D 83$

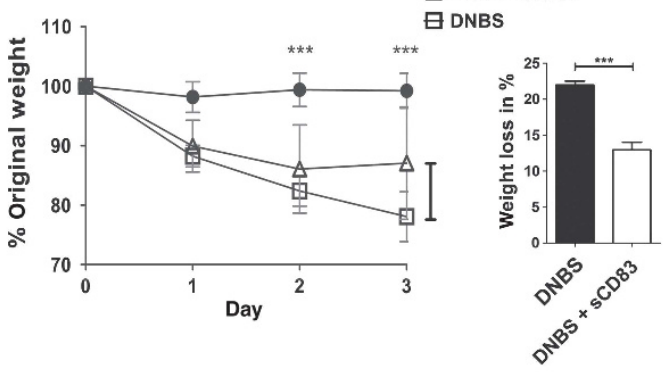

d

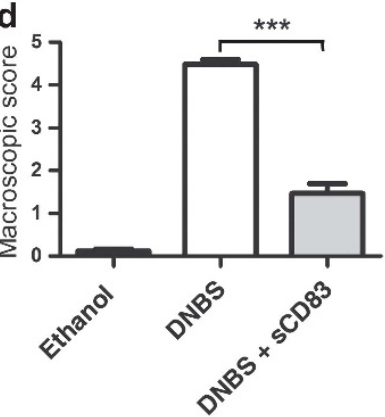

e

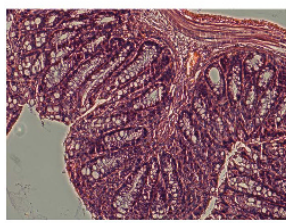

Ethanol

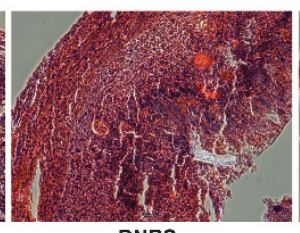

DNBS

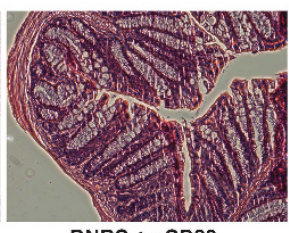

DNBS + sCD83

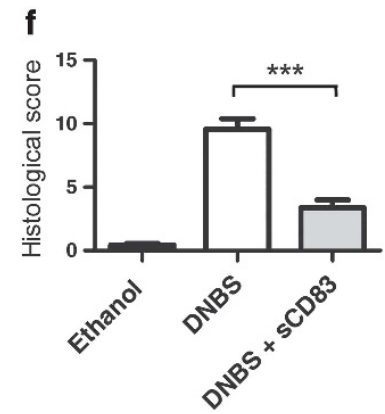

Figure 3 Soluble CD83 (sCD83) ameliorated 2,4-dinitrobenzene sulfonic acid (DNBS)-induced colitis. Inflammation was assessed in DNBS-treated Balb/c mice by (a) survival, $n=55-75$ mice/group. (b, left panel) Weight loss $72 \mathrm{~h}$ after treatment. (b, right panel) Weight loss at day 3, $n=57-80$ mice/group. (c) Colon length, upper: DNBS + sCD83, middle: DNBS only, bottom: ethanol control. Bar $=1 \mathrm{~cm}$. (d) Macroscopic disease score, $n=55-75$ mice/group. (e, f) Histological damage; $n=18-27$ mice/group. Mean \pm s.e.m., ${ }^{\star * \star} P<0.001$, analysis of variance (ANOVA) Tukey's multiple comparison test.

In our experiments, DNBS-induced colitis was associated with increased expression of the inflammatory cytokines TNF- $\alpha$, IL-1 $\beta$, IL-6, IL-17, and mediators as arginase-1, cyclooxygenase 2, and inducible nitric oxide synthase (Figure 4). There was also an increased IFN- $\gamma$ expression in the colons of mice treated with only DNBS (Figure 4). As reaction to the severe tissue damage and oxidative stress in DNBS mice, cytochrome P450 superfamily members Cyp1A1 and Cyp1B1 were also increased. Interestingly, there was a strong and significant inhibition of these inflammatory mediators in the colons of sCD83-treated DNBS mice (Figure 4). On day 3 , all mRNA levels were similar to those detected in ethanoltreated control animals. No significant changes in Th2 cytokine mRNA levels (GATA 3, IL-4, and IL-10) or upregulation of regulatory T cell (Treg)-specific marker genes (Foxp3, TGF- $\beta$ ) could be observed in DNBS $+\mathrm{sCD} 83$-treated mice (Figure 4, third row). Foxp3 mRNA levels of DNBS + sCD83-treated mice mimic those of control animals as confirmed by additional experiments (Supplementary Figure S3A). The thymic stromal lymphopoietin (TSLP) levels were significantly higher in the DNBS + sCD83-treated group as compared with DNBSonly-treated mice and resembled the expression levels of ethanol-treated controls (Figure 4). TSLP is produced by epithelial cells, keratinocytes, and DCs of the intestine. It protects against colitis by limiting Th17 cells and fostering Treg development, ${ }^{21}$ and mediates recovery from colonic inflammation. ${ }^{22}$ Here, sCD83 does not seem to induce TSLP expression, but by blocking inflammation-mediated destruction of the epithelial barrier it may prevent reduction of TSLP expression.

Consistent with histology and pathology, in sCD83-treated animals, no increased infiltration of CD11b-expressing cells was observed when compared with ethanol-treated control animals (Figure 4, bottom row). 

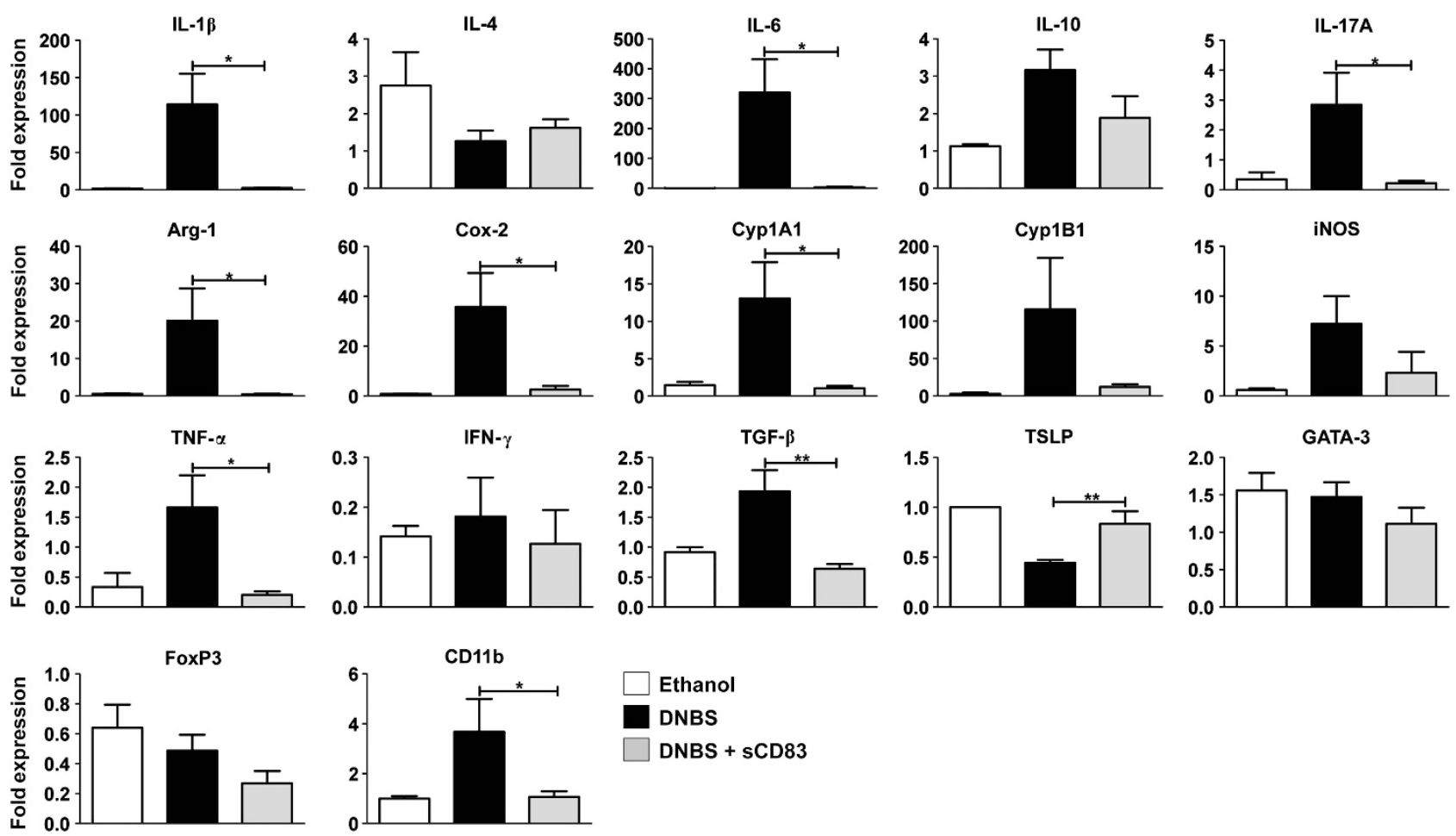

Figure 4 Soluble CD83 (sCD83) treatment abrogates expression of inflammatory mediators and infiltration of macrophages and neutrophils in colons of 2,4-dinitrobenzene sulfonic acid (DNBS)-treated mice. Quantitative real-time reverse-transcriptase-PCR (qRT-PCR) for interleukin (IL)-1 $\beta$, IL-4, IL-6, IL-10, IL-17A, arginase 1 (Arg-1), cyclooxygenase 2 (Cox-2), Cyp1A1, Cyp1B1, inducible nitric oxide synthase (iNOS), tumor necrosis factor- $\alpha$ (TNF- $\alpha$ ), interferon- $\gamma(\mathrm{IFN}-\gamma)$, transforming growth factor- $\beta$ (TGF- $\beta$ ), thymic stromal lymphopoietin (TSLP), GATA3, Foxp3, and CD11b expression on colonic tissue after DNBS colitis on day 3. Mean \pm s.e.m., $n=5-10$ mice/group, data normalized to HPRT; control (black bars), DNBS (open bars), DNBS + sCD83 (gray bars); ${ }^{*} P<0.05,{ }^{* *} P<0.01$, Student's $t$-test.

\section{IDO expression in MLNs was enhanced by SCD83 during DNBS colitis}

To gain further insights, cell subpopulations present in MLNs were analyzed by flow cytometry. Reduced numbers of $\mathrm{CD} 3{ }^{+} /$ $\mathrm{CD}^{+}{ }^{+}$and $\mathrm{CD} 3{ }^{+} / \mathrm{CD} 8{ }^{+}$T cells were observed in DNBS-onlytreated mice that showed a severe colitis on day 3. In contrast, T-cell numbers in MLNs of sCD83-treated DNBS mice were comparable to those found in ethanol-treated control animals (Figure 5a). No significant differences were observed regarding the numbers of $\mathrm{CD} 3{ }^{+} / \mathrm{CD} 4{ }^{+} /$Foxp $^{+}$Tregs (Figure 5a). Levels of $\mathrm{CD}_{11 \mathrm{~b}^{+}}$cells were again elevated in DNBS-treated mice (Figure 5a).

Additional CD3e-specific quantitative real-time reversetranscriptase-PCR (qRT-PCR) data confirmed that emigration of T cells was ameliorated in DNBS + sCD83-treated groups when compared with DNBS-only-treated animals (Figure 5b). In addition, DNBS + sCD83-treated mice matched the ethanol-treated mice regarding their higher CD83 and IFN- $\gamma$ expression in the MLNs (Figure 5b). There were also comparable arginase-1, cyclooxygenase 2 , and inducible nitric oxide synthase expression levels in the MLNs of DNBS + sCD83 mice and ethanol controls (Figure 5b). Inflammatory cytokines such as IL-1 $\beta$, IL-6, IL-17A, and IL-17F were also reduced in DNBS + sCD83-treated- mice when compared with DNBS-only-treated mice (Figure 5b). Thus, sCD83 reduces
T-cell activation, resulting in less emigration of activated cells out of MLNs during DNBS colitis. Next to activation, the reduced infiltration of activated leukocytes into the intestine might be because of a defect in cell migration caused by sCD83, and therefore this has been investigated. However, we could not detect different expression levels of the gut-homing receptors CCR9 and of the $\alpha 4$ integrin CD49d in CD83-treated animals (Supplementary Figure S3C). In addition, we studied the capacity of sCD83 to block migration of lipopolysaccharidestimulated bone marrow (BM)-derived BM-DCs against a CCL19 chemokine gradient in vitro. However, as shown in Supplementary Figure S3D, sCD83 did not influence DC migration at all. Another possible explanation for the reduced number of activated cells could be that sCD83 induces apoptosis. Because of inflammation, there will always be a much higher apoptosis rate in tissues of DNBS-only-treated mice in comparison with DNBS + sCD83-treated animals. Therefore, we compared naive sCD83-treated vs. control mice without colitis induction. The mice were injected with $100 \mu \mathrm{g}$ sCD83 intraperitoneally for 3 days and we analyzed their splenocytes on day 4 by flow cytometry (Supplementary Figure S3E). There were no effects on B-cell, T-cell, DC, or CD11b ${ }^{+}$cell homeostasis. Using 7-aminoactinomycin D and Annexin V co-staining of splenocytes also showed no enhanced cell death in sCD83-treated mice in vivo (Supplementary Figure S3E). 


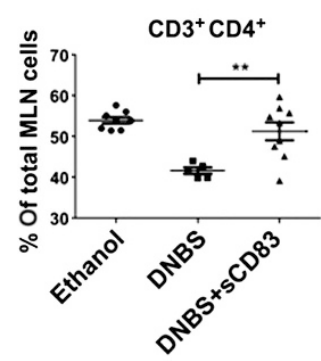

b

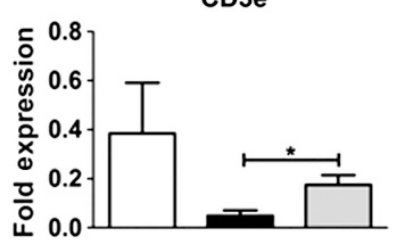

IL-1 $\beta$
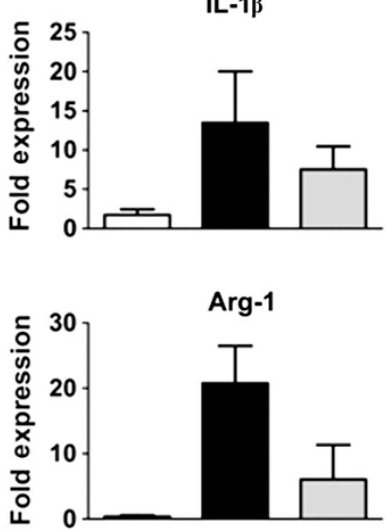

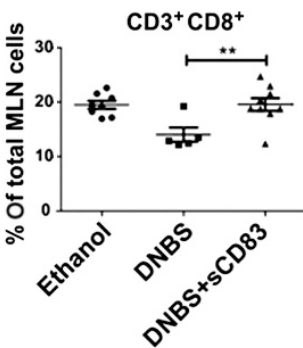

CD83

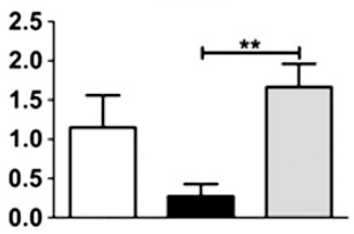

IL-6

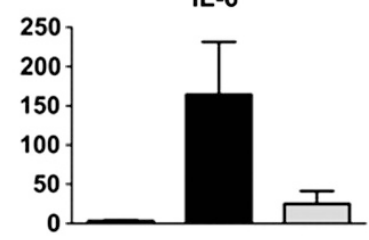

Cox-2

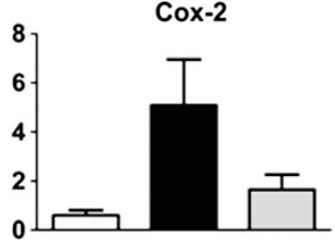

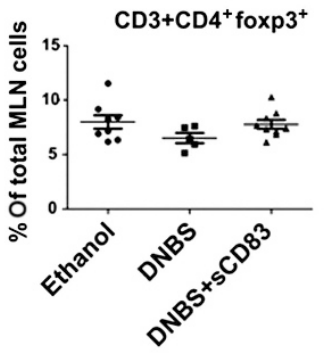

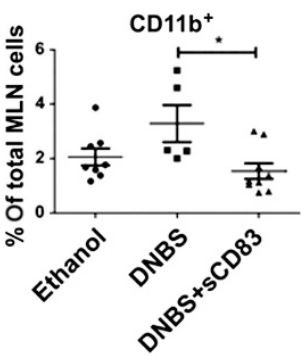

IFN- $\gamma$

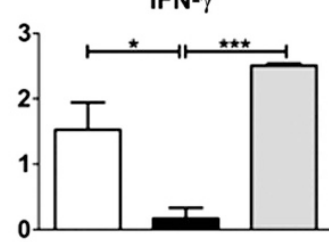

IL-17A
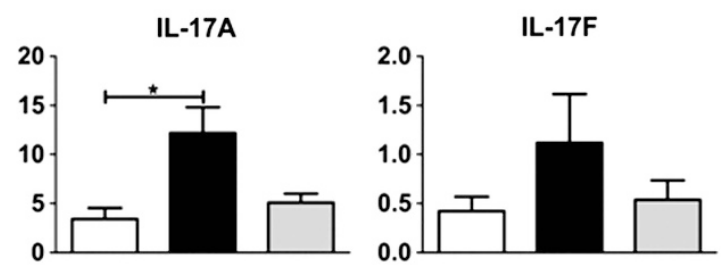

iNOS

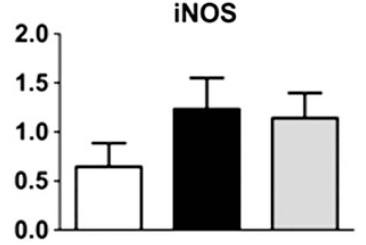

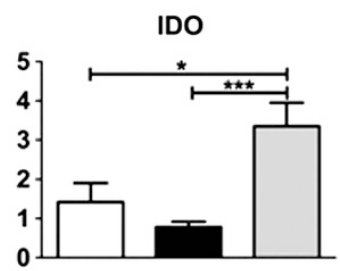

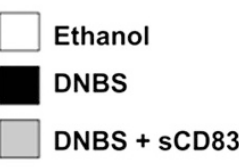

Figure 5 Soluble CD83 (sCD83) normalized T-cell numbers and enhanced indoleamine 2,3-dioxygenase (IDO) expression in mesenteric lymph nodes (MLNs). (a) Flow cytometry of MLNs showed equal numbers of $\mathrm{CD} 3^{+} \mathrm{CD} 4^{+}$and CD3 ${ }^{+} \mathrm{CD} 8^{+} \mathrm{T}$ cells in DNBS + sCD 83 and ethanol mice, no change in $\mathrm{CD}^{+}{ }^{+} \mathrm{CD} 4^{+} \mathrm{Foxp}^{+}$regulatory T cells (Tregs) in all groups, and more CD11b ${ }^{+}$cells in 2,4-dinitrobenzene sulfonic acid (DNBS)-only mice at day $3(n=5-9)$. (b) Quantitative real-time reverse-transcriptase-PCR (qRT-PCR) for CD3e, CD83, interferon- $\gamma$ (IFN- $\gamma$ ), IDO, interleukin (IL)-1 $\beta$, IL-6, IL-17A, IL-17F, arginase 1 (Arg-1), cyclooxygenase 2 (Cox-2), and inducible nitric oxide synthase (iNOS) expression on MLNs after DNBS-induced colitis on day 3 (mean \pm s.e.m., $n=5-11$ mice/group, data normalized to HPRT; control (black bar), DNBS (open bar), DNBS + sCD83 (gray bar); ${ }^{\star} P<0.05,{ }^{* *} P<0.01,{ }^{* *} P<0.001$, Student's $t$-test).

Identical ex vivo flow cytometric analysis of MLN cells revealed comparable results (data not shown). Thus, sCD83 does not induce enhanced cell death of major leukocyte populations. In addition, we examined colons of mice injected with $100 \mu \mathrm{g}$ sCD83 intraperitoneally for 3 days on day 5 . The sCD83-treated mice demonstrated sustained proliferation of colonic cells by Ki67 staining similar to naïve mice and no increased apoptosis by caspase-3 and -8 staining (Supplementary Figure S3F).

It is well known that IDO is involved in immunosuppression and downmodulation of overshooting immune responses in vitro as well as in vivo. ${ }^{23-25}$ Therefore, using qRT-PCR we also analyzed whether IDO expression levels are altered by sCD83 in our intestinal inflammation model. As a matter of fact, we could detect significantly increased IDO mRNA expression levels in the MLNs of DNBS + sCD83-treated animals in comparison with control and DNBS-only-treated mice (Figure 5b). These data indicated that IDO might indeed be functionally relevant for the protective effects observed in sCD83-treated animals.

\section{Inhibition of IDO interferes with SCD83-induced protective effect}

IDO is an enzyme that catalyzes the degradation of the essential amino acid tryptophan in many cell types. The enzymatic activity of IDO can specifically be blocked using the inhibitor $1-\mathrm{MT}{ }^{26}$ Thus, in order to further investigate the role of IDO in connection to sCD83-mediated immunosuppressive effects in our IBD model, in the following in vivo experiments IDO activity was blocked by implanting 1-MT impregnated pellets $\left(15 \mathrm{mg} \mathrm{day}^{-1}\right)$ or vehicle control pellets under the dorsal skin of the neck on day -1 . DNBS was administered on day 0 and mice were treated with sCD83 on days $-1,0$, and +1 . Interestingly, when IDO activity was blocked by $1-\mathrm{MT}$ in DNBS + sCD83-treated animals, the previously observed 


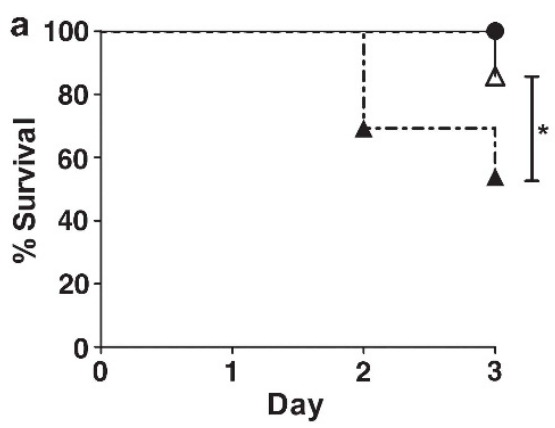

- Ethanol

$\triangle$ DNBS + Placebo + sCD83

- DNBS + 1-MT + sCD83

\section{b}

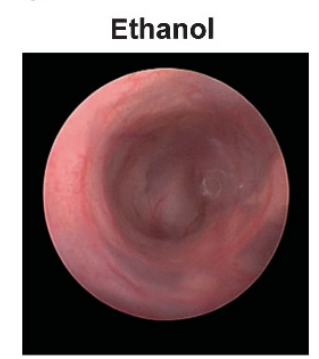

C

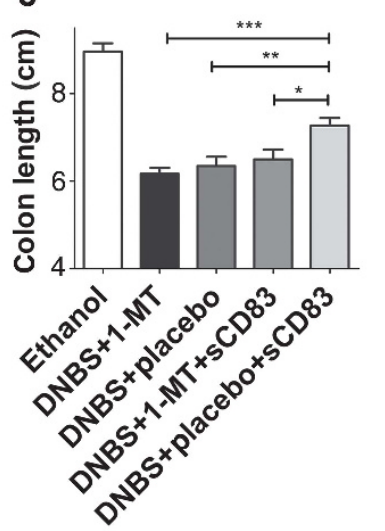

DNBS

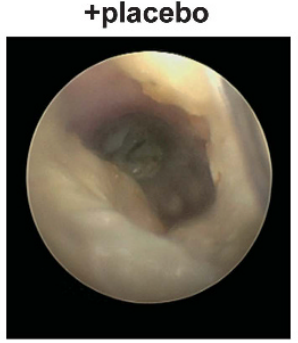

d

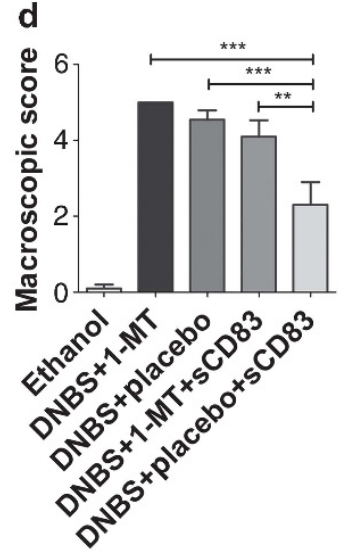

DNBS

+1-MT + sCD83

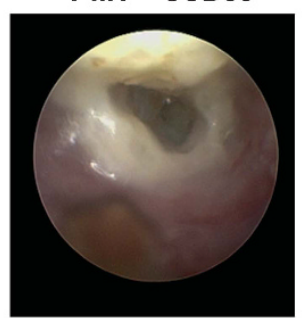

e

Figure 6 Indoleamine 2,3-dioxygenase (IDO) inhibitor 1-methyl-tryptophan (1-MT) diminished the protective effects of soluble CD83 (sCD83) in 2,4dinitrobenzene sulfonic acid (DNBS) colitis. SCD83- or control-treated DNBS-induced colitis mice were preimplanted with 1-MT impregnated tablets or vehicle (placebo) and monitored from day 0 to day 3 for (a) mortality. (b) Endoscopic pictures of ethanol control, DNBS + placebo, DNBS +1 -

$\mathrm{MT}+\mathrm{sCD} 83$, and DNBS + placebo + sCD83 colon. (c) Colon length. (d) Macroscopic score. (e) Histological score. (a) $n=15$ mice/group; ${ }^{* *} P<0.01$, Mantel-Cox test; (c-e) mean \pm s.e.m., $n=$ minimum 10 mice/group; ${ }^{*} P<0.05,{ }^{\star \star} P<0.01,{ }^{\star \star \star} P<0.001$, analysis of variance (ANOVA) Tukey's multiple comparison test.

sCD83-mediated protective effects were lost (see Figure 6a and Supplementary Figure S4A). In contrast, DNBS+ sCD83-treated animals that were implanted with placebo pellets showed the previously observed protective sCD83 effects.

Standard endoscopy analyses showed nontransparent colons and extreme fibrin cover in DNBS + placebo- and DNBS + 1$\mathrm{MT}+$ sCD83-treated animals (Figure 6b). In contrast, in $\mathrm{DNBS}+$ placebo + sCD83-treated animals, transparent and only moderate thickening of colon was observed (Figure $6 \mathbf{b}$ ), comparable with ethanol-treated control animals (Figure 6b). In addition, when colon length (Figure 6c), weight loss, (Supplementary Figure S4C) and the overall macroscopic and histological score (Figure 6d, e) was used as readout, again a loss of the $\mathrm{sCD} 83$-induced therapeutic effect could be observed in 1-MT-treated animals. Thus, IDO is critically involved in sCD83-mediated immunomodulatory effects and the block of IDO activity increased DNBS-induced mortality and clinical symptoms in sCD83-treated mice.

\section{DISCUSSION}

The etiology of IBD is still not completely understood. Decades of research revealed that it must be a multifactorial disorder. Current therapies combine anti-inflammatory drugs and agents that reduce IBD-associated symptoms. However, development of steroid dependency is quite common in these patients. Anti-TNF- $\alpha$, one of the new biological drugs, targets the inflammatory cytokines directly, but it also implies the risk 
of toxic side effects. ${ }^{2,3,5,27}$ Another discussed approach besides antibody-based immunosuppressive strategies is the use of physiologic immune modulators, e.g., anti-inflammatory neuropeptides, such as the vasoactive intestinal peptide that regulates immune tolerance. ${ }^{28}$ Vasoactive intestinal peptide has been shown to have a physiologic homeostatic role in TNBSinduced colitis ${ }^{29}$ and has provided therapeutic effects in this disease model. ${ }^{30}$

Here, we analyzed sCD83, a new physiologic immune mediator, ${ }^{8,9}$ that showed highly promising therapeutic properties in inflammatory diseases. Several studies elucidated that $\mathrm{SCD} 83$ has inhibitory function on DC and T cellmediated activation of the immune system. ${ }^{12-17,31,32}$ In this respect, sCD 83 could prevent the acute autoimmune symptoms of experimental autoimmune encephalomyelitis, a murine model for the early inflammatory phase of human multiple sclerosis. ${ }^{15}$ In addition, it induced allograft tolerance in murine kidney, ${ }^{14,17}$ skin, ${ }^{16}$ and heart ${ }^{17}$ transplantation studies. The molecule SCD83 is a physiologic mediator, as it is also present in sera of healthy individuals. ${ }^{8}$ In addition, it is part of an immune escape mechanism of viruses ${ }^{11}$ and tumors. ${ }^{9}$

When this study was initiated, it was not known whether CD83 is also expressed in the GI tract under physiologic conditions. Using our CD83eGFP reporter mouse system, ${ }^{18}$ we could show for the first time that the CD83 promoter is highly active in MLNs, Peyer's patches, and isolated lymphatic follicles in the GI tract comparable to high expression in other lymphatic organs such as spleen and peripheral lymph nodes (Figure $\mathbf{1 b}, \mathbf{c}$ ). The major $\mathrm{CD} 83^{+}$populations were identified as $\mathrm{B}$ cells followed by $\mathrm{CD} 3^{+} / \mathrm{CD} 4^{+}$and $\mathrm{CD} 3^{+} / \mathrm{CD} 8^{+}$ $\mathrm{T}$ lymphocytes (Figure 1b,d). Among the myeloid subpopulations, CD83 was mainly expressed by $\mathrm{CD}_{11 \mathrm{c}^{+}}$DCs and $\mathrm{Ly}_{6 \mathrm{C}}{ }^{+}$inflammatory monocytes as well as $\mathrm{F} 4 / 80^{+}$ macrophages (Figure 1b,d). Detailed investigation of LPinfiltrating cells and inflammatory conditions showed that next to the major B-cell population (70-80\%) a minor $\mathrm{CD} 3{ }^{+} \mathrm{T}$-cell population $(\sim 6 \%)$ was present. Noteworthy, a surprisingly high DN T-cell population (29\%) was found to express CD83 within the $\mathrm{CD}^{+}$T-cell population (Supplementary Figure S1D). Next to DCs (11\% of CD $83^{+}$cells), we also identified $\mathrm{CD} 3^{+}$eosinophils $(50 \%)$ as well as monocyte/macrophages (38\%), but only a small population of neutrophils (2\%) within the $\mathrm{CD} 11 \mathrm{~b}^{+}$cell population in the LP (Supplementary Figure S1E, F). Interestingly, in pilot studies on intestinal tissues of Crohn's patients, we also detected increased CD83 mRNA levels in direct relation to inflammatory cytokine mRNAs such as IFN- $\gamma$ (data not shown).

Another striking finding of this study was the massive infiltration of $\mathrm{CD} 83^{+}$leukocytes into inflamed colonic tissues in DNBS-treated mice (Figure 2). As it has previously been reported that $\mathrm{CD} 83^{+}$cells are able to shed an immunosuppressive soluble form of $\mathrm{CD} 833^{8,9,11}$ we next analyzed whether this is also the case in the colon. Especially in DNBStreated animals, we detected increased levels of sCD83 in colonderived cells (Figure 2f).
Thus, one could hypothesize that immunosuppressive endogenous sCD83 might play a role in the control of IBD. However, in severe colitis the amounts necessary to substantially suppress the development of the disease might not be high enough. Hence in future studies, it would be a promising approach to investigate possible ways to increase the release of sCD83 by infiltrating $\mathrm{CD} 83^{+}$cells of inflamed tissues in vivo. However, the mechanisms of $\mathrm{sCD} 83$ release are still ambiguous. Thus, the therapeutic application of sCD83 in concentrations that are high enough to suppress IBD-related disease symptoms is a realistic and interesting option. To test this hypothesis in vivo, we used the well-characterized DNBS-induced colitis model that has frequently been used to study the mechanisms of colonic inflammation and to investigate new anti-inflammatory components. ${ }^{33-35}$ This model resembles human IBD including strong infiltration of colonic mucosa by neutrophils and macrophages. There is also increased production of inflammatory mediators including Th1 cytokines. Hence, DNBS introduced into the colon induces a $\mathrm{T}$ cell-mediated immune response leading to dense infiltration of activated $\mathrm{T}$ cells, macrophages, and neutrophils into the large bowel wall. ${ }^{20}$

Mortality is a very clear clinical end point in order to investigate the efficacy of a new therapeutic agent. As shown in the Result section, the administration of sCD83 dramatically reduced the mortality rate to $<3 \%$ (Figure $3 \mathbf{a}$ ). In addition, weight kinetics clearly showed that DNBS + sCD83-treated mice started to recover already between days 2 and 3 after DNBS treatment, whereas DNBS-only-treated mice still lost weight on day 3 (Figure 3b). Remarkably, there was an over $40 \%$ reduced weight loss in DNBS + sCD83-treated animals when compared with DNBS-only-treated mice at day 3 (Figure 3b). The improvement of the macroscopic score on day 3 after DNBS application was highly significant in SCD83treated animals (Figure 3d). Histological evaluation of the colon 3 days after DNBS administration revealed significantly decreased granulocyte infiltrates in mucosa and submucosa, as well as reduced thickening of the colonic wall and absence of goblet cell depletion in DNBS + sCD83-treated animals (Figure 3e,f).

In particular, cytokine imbalance and the production of inflammatory mediators, mainly TNF- $\alpha$, have been implicated to be crucial in experimental DNBS colitis and human IBD. ${ }^{36}$ $\mathrm{T}$ cell-derived cytokines, such as IL-2, IFN- $\gamma$, and TNF- $\alpha$, perpetuate gut inflammation by activating tissue macrophages and granulocytes to release proinflammatory cytokines and mediators, including IL-1 $\beta$, IL-6, IL-12, nitric oxide, and TNF- $\alpha .{ }^{3}$ Hence, we investigated the expression of cytokines by qRT-PCR in colonic tissues. There was a clear reduction of TNF- $\alpha$, IL-1 $\beta$, IL- 6 , and IL-17A and also a reduced IFN- $\gamma$ expression in the colons of DNBS + sCD83-treated mice compared with DNBS-only-treated animals (Figure 4). Overall, sCD83 treatment strongly reduced expression of inflammatory cytokines as well as other proinflammatory mediators including arginase- 1 , cyclooxygenase 2 , and inducible nitric oxide synthase that are typical markers for activated macrophages and neutrophils in colonic tissues (Figure 4). 
This correlated with a reduced number of CD11b-positive cells after sCD83 treatment (Figure 4). Further immunofluorescence analyses using MELC identified these CD11b-positve cells as $\mathrm{CD} 11 \mathrm{~b}^{+} / \mathrm{Ly}_{6 \mathrm{G}}{ }^{+}$neutrophil granulocytes (yellow) and

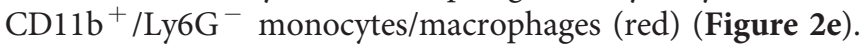
The massive infiltration of neutrophil granulocytes and monocytes/macrophages into colonic tissue, as demonstrated by hematoxylin and eosin staining and immunofluorescence microscopy, on day 3 after DNBS-induced colitis, was clearly prevented by sCD 83 treatment (Figures $3 e, \mathbf{f}$ and 4 ).

In MLNs, treatment with sCD83 restored cellular distributions that are typical for healthy mice (Figure 5a). Furthermore, sCD83 induced significantly increased expression levels of IDO in the MLNs (Figure 5b). IDO expression can be induced by IFN- $\gamma$ and represents the first enzyme in the kynurenine pathway and catabolizes the generation of $\mathrm{N}$-formylkynurenine from the amino acid L-tryptophan. Depletion of L-tryptophan leads to an antiproliferative effect in target cells, and thus IDO has been reported to be the key enzyme in the inhibition of T-cell proliferation by IFN- $\gamma$-stimulated DCs and macrophages. ${ }^{23,24,37}$

Interestingly, IFN- $\gamma$ expression levels in MLNs of DNBS + sCD83-treated mice are comparable to those found in ethanoltreated control animals (see Figure 5). The elevated IFN- $\gamma$ expression levels as compared with that in DNBS-only-treated animals could be explained by the following hypothesis: SCD83 treatment leads to a reduced infiltration of $\mathrm{CD}_{11} \mathrm{~b}^{+}$cells (see Figure 5a) and a strong reduction of inflammatory cytokines (IL-1 $\beta$, IL-6, IL-17A, and IL-17F; see Figure 5b) that are necessary to induce complete T-cell activation in MLNs and subsequently emigration of $\mathrm{T}$ cells. This is also reflected by the reduced T-cell numbers in MLNs derived from DNBS-onlytreated animals (see Figure 5a). In contrast, DNBS + sCD83treated animals show no decreased levels of $\mathrm{T}$ cells compared with controls. This could possibly explain why IFN- $\gamma$, which is mainly expressed by activated T cells, is expressed at higher levels in sCD83-treated animals, comparable to levels observed in ethanol-treated control mice. In addition, these IFN- $\gamma$ levels could subsequently lead to the induction of IDO as discussed above. However, the precise mechanism(s) have to be investigated in further studies.

To further substantiate the crucial role of IDO in sCD83mediated immunosuppression, 1-MT, a tryptophan analog and pharmacological inhibitor of IDO, was used. Strikingly, the systemic administration of 1-MT abrogated the protective effect of sCD83 in our experimental colitis model (Figure 6a-e and Supplementary Figure S4). These results correlate very well with kidney allograft transplantation experiments where sCD83 has been shown to induce tolerogenic DCs in an IDOdependent manner. ${ }^{14}$ In this respect, it has recently been reported that in DCs, in addition to its enzymatic properties, IDO also possesses a signal transducing activity and induces in a TGF- $\beta$-dependent manner long-term tolerance by tolerogenic DCs and Tregs. ${ }^{25,38}$

However, in the inflammatory colitis model used here, sCD83 did not lead to the expansion of Tregs, but instead blocked the infiltration of inflammatory macrophages and granulocytes. Thus, in this short-term acute colitis model, induction of tolerance might not be the key element. In addition, we could not detect increased TGF- $\beta$ and/or Foxp3 expression levels in sCD83-treated protected mice (Figures 4 and 5a and Supplementary Figure S3). Instead, we observed comparable IFN- $\gamma$ expression levels as in ethanol control mice. IFN- $\gamma$ has been reported to be connected to short-term IDOinduced immunosuppressive activities, whereas the long-term effects are TGF- $\beta$ dependent. ${ }^{25}$ In addition, Gurtner et al. showed that IDO is expressed by antigen-presenting cells in the LP at baseline and is induced in multiple cells by rIFN- $\gamma$. In their study, IDO inhibition by 1-MT resulted in a more severe TNBS colitis. ${ }^{39}$ In accordance with this study, inhibition of IDO alone also increased the severity of colitis in our DNBS-treated mice (Figure 6 and Supplementary Figure S4).

In addition, very recent studies showed that neutrophils undergo apoptosis in the presence of kynurine and absence of tryptophan. Van der Sluijs et al. ${ }^{40}$ proposed that pulmonary IDO activity, catalyzing tryptophan degradation, is responsible for neutrophil apoptosis during influenza infection. Also, we did see strongly reduced expression of arginase-1, cyclooxygenase 2, and inducible nitric oxide synthase that is mainly expressed in proinflammatory neutrophils and macrophages (Figure 4). This could at least partially explain the strong protective effect of sCD83 in this mainly neutrophil-driven acute colitis model. Nevertheless, we observed no change in the balance of leukocyte populations and no enhanced apoptosis of leukocytes by sCD83 without inflammation (Supplementary Figure S3E). Thus, sCD83 might block only in the acute phase of inflammation without a general apoptotic effect on naive leukocytes.

In addition, no sCD83 impact on expression levels of gut-homing receptor CCR9 and $\alpha 4$ integrin CD49d (Supplementary Figure S3C) were detectable. Thus, sCD83 has most likely no direct influence on cell migration.

In summary, here we provide the first proof of principle for the therapeutic effect of sCD83 in experimental colitis. Our results demonstrate that sCD83 treatment of mice effectively reduces the severity of DNBS-induced colitis as shown by a drastically reduced mortality rate, inhibition of proinflammatory cytokine expression, and decreased infiltration of inflammatory granulocytes and macrophages into the GI tract. Thus, sCD83 opens new and promising therapeutic options and has the potential to be further explored in future studies.

\section{METHODS}

Mice. CD83eGFP knock-in mice were bred in the Franz-PenzoltZentrum (Erlangen, Germany). This reporter mouse strain was described previously. ${ }^{18}$ BALB/c mice were purchased from Charles River Laboratories, Sulzfeld, Germany. The institutional and national guide for the care and use of laboratory animals was followed.

Experimental colitis. Colitis was induced by intrarectal instillation of $4 \mathrm{mg}$ of DNBS (Sigma, Deisenhofen, Germany) in $100 \mu \mathrm{l}$ of a 1:1 ethanol/phosphate-buffered saline (PBS) solution via a $4-\mathrm{cm}$ catheter in BALB/c mice. Mice receiving 50\% ethanol alone or PBS only served as time-matched controls. Mice were killed at 3 days after treatment. 
Colitis was assessed by clinical disease scores $($ maximum $=5)$ based on the presence (1) or absence ( 0 ) of the following: $>10 \%$ weight loss, wet anus/soft stool/empty colon, shortening of the colon, anal bleeding/ occult blood, macroscopic ulcers, and death. Histological damage scores were determined on hematoxylin and eosin-stained sections (formalin-fixed, paraffin-embedded tissues; $3 \mu \mathrm{m}$ sections) and are based on the following score: loss of architecture, $0-3$; inflammatory infiltrate, $0-3$; goblet cell depletion, 0 or 1 ; ulceration, 0 or 1 ; edema, 0 or 1; muscle thickening, $0-2$; and presence of crypt abscesses, 0 or 1 (maximum score $=12$ ) as published previously. ${ }^{41}$

Isolation of LP mononuclear cells from murine colonic tissue. To isolate mononuclear cells from intestinal LP, isolated mouse colons of killed mice were dissociated combining mechanical dissociation with enzymatic degradation using the Lamina Propria Dissociation Kit mouse (Miltenyi Biotec, Bergisch Gladbach, Germany) according to the manufacturer's protocol. In short, first the intraepithelial lymphocytes were disrupted from mucosa by shaking the tissue in $1 \times$ Hanks' balanced salt solution (w/o) containing 5 mM EDTA, 5\% fetal bovine serum, and $1 \mathrm{~mm}$ dithiothreitol. Then, the LP tissue was enzymatically and mechanically dissociated into a single-cell suspension using the gentleMACSTM Dissociators (Miltenyi Biotec). In addition to the manufacturer's protocol, the resulting cells were further purified using a Percoll gradient $(40 \% / 80 \%)$.

Immunosuppression. The extracellular domain of human CD83 (amino acids 20-145), sCD83, was purified from bacteria before intraperitoneal injection of $100 \mu \mathrm{g} s \mathrm{SD} 83$ in $100 \mu \mathrm{l}$ saline solution ( $>50$ endotoxin units/mg protein) $;{ }^{42}$ at $-1,0$, and +1 days to DNBS instillation.

IDO inhibition by 1-MT pellet implantation. Slow-release polymer pellets (IRA, Sarasota, FL) impregnated with 1-MT (15 mg/day for 14 days) or placebo pellets were inserted surgically under the dorsal skin between ear and shoulder 1 day before the intrarectal administration of DNBS (day - 1).

qRT-PCR. Tissues were removed and stored in RNAlater solution (Life Technologies, Darmstadt, Germany) at $-80^{\circ} \mathrm{C}$. Total RNA was prepared from tissue using the RNeasy Plus Mini Kit (Qiagen, Hilden, Germany). Traces of genomic DNA were removed by DNase digestion with the RNase-free DNase Set (Qiagen). Subsequently, cDNA synthesis was performed (First Strand cDNA Synthesis Kit; Thermo Scientific, Schwerte, Germany). Primers selected from a primerbank or previously published primers (Supplementary Table S1) were used in qRT-PCR with the DyNAmoTM Capillary SYBR Green qPCR Kit (Finnzymes, Espoo, Finland). Relative Quantification normalizing to HPRT was performed using the Light Cycler Software (Roche, Mannheim, Germany).

High-resolution colonoscopy. Endoscopy in live mice has been performed as previously described. ${ }^{43}$

Immunohistochemistry. Tissues were formalin fixed and processed according to standard histological protocols. Staining with $A B C$ reagent was conducted according to the manufacturer's instructions (Vector Laboratories, Peterborough, UK). Sections were stained for CD83 (AF-1437 (R\&D, Wiesbaden, Germany) and rabbit anti-goat IgG biotin (Dako Cytomation, Hamburg, Germany)) and CD8 (eBioH35 (eBioscience, Frankfurt, Germany) and goat anti-rat IgG biotin (Pharmingen, Heidelberg, Germany)) with a standard protocol for development with AEC substrate. Tissues were counterstained with haemalaun (Merck, Darmstadt, Germany). In some samples, immunofluorescence of tissue sections was performed using the TSC Cy3 system as recommended by the manufacturer (PerkinElmer, Hamburg, Germany) and the following antibodies were used: the primary antibodies anti-activated caspase-3 (polyclonal Cat. no. 9661, Cell Signaling, Frankfurt, Germany), anti-activated caspase-8 (D5B2 Cat. no. 8592, Cell Signaling), anti-Ki67 (SP6 Cat. no. ab16667, Abcam,
Cambridge, UK), and a biotinylated secondary anti-rabbit-antibody (goat anti-rabbit IgG-B: sc-2040, Santa Cruz, Heidelberg, Germany). The nuclei were counterstained with Hoechst 33342 (Invitrogen, Darmstadt, Germany). Fluorescence microscopy (Leica, Wetzlar, Germany) was used for analysis.

ELISA. Colons were dissociated mechanically. Colonic single-cell suspensions of $10^{5}$ cells were incubated in $100 \mu \mathrm{RPMI}$ for $6 \mathrm{~h}$ at $37^{\circ} \mathrm{C}$ in a $\mathrm{CO}_{2}$ incubator. sCD83 ELISA was conducted in a sCD83 sandwich enzyme immunoassay kit (Uscn Life Science, Wuhan, China) according to the protocol of the manufacturer.

Flow cytometry. Colons and MLNs were dissociated mechanically. Cell surface staining using flow cytometry employed the standard procedures and the following conjugated antibodies: anti-B220 (RA36B2), anti-CD3 (500A2), anti-CD4 (RM4-5), anti-CD11b (M1/70), anti-CD11c (HL3), anti-CD83 (Michel-19), anti-CD103 (M290), antiLy-6G (1A8) (BD Pharmingen, Heidelberg, Germany); anti-CD8 (536.7), anti-Ly6C (HK1.4) (Biolegend, London, UK); and anti-F4/80 (BM8) (eBioscience). Intracellular staining for Foxp3 (FJK-16s) was conducted according to the manufacturers' instructions (eBioscience). Colon and MLN single-cell suspensions were incubated with appropriate fluorochrome-conjugated monoclonal antibodies and run for 4- or 7-color fluorescence staining on a cytofluorometer (FACS Canto II, BD, Heidelberg, Germany) and analyzed with FlowJo 10 (Tree star, Ashland, OR).

Multiepitope ligand cartography. Cryostat tissue sections $(5 \mu \mathrm{m})$ were performed from colon using a cryotome (Leica CM 3050S). Samples were incubated in acetone for $10 \mathrm{~s}$, air dried, and kept on storage at $-20{ }^{\circ} \mathrm{C}$ for several days. In immediate preparation for subsequent MELC analysis, the samples were incubated in acetone for $10 \mathrm{~min}$ at $-20^{\circ} \mathrm{C}$ and then air dried for $10 \mathrm{~min}$ at room temperature. For rehydration the sections were incubated with PBS pH 7.4 (Lonza, Verviers, Belgium) for $5 \mathrm{~min}$ and then rinsed five times in PBS. Afterwards, the samples were incubated in normal goat serum (Dako, Hamburg, Germany, 1:30 in PBS) for $30 \mathrm{~min}$ and then washed five times with PBS. For the MELC measurement, a slide with a colon sample was placed on the stage of a fluorescence microscope as part of the MELC robot technology. ${ }^{44,45}$ Then, fluorescein isothiocyanate (FITC)-labeled antibodies (anti-Ly6G, clone 1A8; anti-CD11b, clone M1/70; anti-CD31, clone 390, all provided by BD Biosciences, Franklin Lakes, NJ) and washing solution (PBS; Lonza) were added and removed under temperature control by the MELC robot. The phase contrast and fluorescence images were acquired by an inverted wide-field fluorescence microscope (Leica DM IRE2; $\times 20$ air lens; numerical aperature 0.7) with a cooled CCD camera (Apogee KX4, Apogee Instruments, Roseville, CA), followed by soft bleaching (centered at $488 \mathrm{~nm}$ for FITC). Recording of all image data and coordination of all system components were controlled by software developed by MelTec $\mathrm{GmbH}$ (Magdeburg, Germany). All these processes (antibody binding/ fluorescence detection/soft bleaching) were part of a fully automated cycle. As a control for unspecific tag-binding, the first MELC cycles were performed with FITC-labeled mouse immunoglobulin G in different dilutions. For MELC data analysis, the fluorescence images produced for each tag binding site were aligned pixel-wise using the corresponding phase contrast images. Flat-field corrections were used to correct the images for illumination.

Generation of BM-DCs. DCs were generated from BM cells derived from $\mathrm{Balb} / \mathrm{c}$ mice. After removing all muscle tissues with gauze from the femurs and tibias, the bones were placed in a $60-\mathrm{mm}$ dish with $70 \%$ ethanol for $1 \mathrm{~min}$ and then rinsed with PBS, and transferred into a fresh dish. Both ends of the bones were cut with scissors and the marrow was flushed out using $\sim 20 \mathrm{ml}$ PBS with a syringe and 25-gauge needle. The BM was suspended and passed through a $70 \mu \mathrm{m}$ cell strainer to remove small pieces of bone and debris. After washing, the cells were diluted 1:1000 in Casyton and counted in the CASY Cell Counter 
(Schärfe System, Reutlingen, Germany). $2 \times 10^{6}$ fresh BM cells were cultured in $10 \mathrm{ml} \mathrm{R} 10$ medium with $10 \%$ culture supernatant of a murine granulocyte macrophage colony-stimulating factor (GM-CSF)-transfected cell line (equivalent to $>200 \mathrm{U} \mathrm{ml}^{-1}$ ). At day $3,10 \mathrm{ml}$ of fresh medium $(\mathrm{R} 10+10 \%$ GM-CSF $)$ was added. At days 6 and $8,10 \mathrm{ml}$ medium was removed and replaced with $10 \mathrm{ml}$ fresh medium $(\mathrm{R} 10+10 \%$ GM-CSF). Mature DCs were generated by adding lipopolysaccharide ( $100 \mathrm{ng} \mathrm{ml}^{-1}$; Sigma-Aldrich) overnight to day 8 BM cultures. The BM-DCs were harvested at day 9 and counted in the CASY Cell Counter.

DC migration assay. Lipopolysaccharide-stimulated BM-DCs were resuspended in migration medium to a final cell number of $2 \times 10^{6} / \mathrm{ml}$. In each of the 24 wells of the transwell plate (Corning Costar, London, UK), $600 \mu \mathrm{l}$ migration medium per well was added. Subsequently, preequilibrated transwell inserts with a pore size of $5 \mu \mathrm{m}$ were added to the wells. $2 \times 10^{5}$ cells $(100 \mu \mathrm{l})$ were seeded into the inserts. To specific ones, 25 or $50 \mu \mathrm{g} \mathrm{ml}^{-1} \mathrm{sCD} 83$ were added. In addition, cells were also put aside for the standard curve. In order to analyze the migration toward the chemokine gradient, CCL19 (100 $\mathrm{ng} \mathrm{ml}^{-1}$, Peprotech, Hamburg, Germany) was added to the lower compartment. Cells were allowed to migrate for $2 \mathrm{~h}$ at $37^{\circ} \mathrm{C}$. Afterward, DCs were harvested from the lower chamber and the cell numbers were determined by measuring the $\beta$ glucuronidase activity of migrated cells as compared with the standard curve as described below.

$\beta$-Glucoronidase assay. To determine the numbers of migrated BM-DCs, cells were resuspended in $25 \mu \mathrm{l}$ PBS and $5 \mu \mathrm{l}$ 1\% TritonX-100 in PBS was added. After 15 min of incubation at room temperature, the debris was removed by centrifugation and $25 \mu$ lof the respective lysates were incubated with $75 \mu \mathrm{l} 10 \mathrm{~mm}$ 4-nitrophenyl- $\beta$-D-glucuronide (NPDG) solved in $100 \mathrm{~mm}$ sodium acetate, $\mathrm{pH} 4$, for $2 \mathrm{~h}$ at $37^{\circ} \mathrm{C}$. Subsequently, $100 \mu \mathrm{l} 0.4 \mathrm{M}$ glycin, $\mathrm{pH} 10$, was added and the absorbance at $405 \mathrm{~nm}$ was measured using a Victor2 multilabel counter (PerkinElmer, Jugesheim, Germany).

Statistics and data presentation. All statistics were calculated in Prism 5.0 (GraphPad, La Jolla, CA) and are analysis of variance with Tukey's post-test or Student's $t$-test as indicated in the respective figure legends. All data are presented as mean \pm s.e.m.

SUPPLEMENTARY MATERIAL is linked to the online version of the paper at http://www.nature.com/mi

\section{ACKNOWLEDGMENTS}

This work was supported by the Deutsche Forschungsgemeinschaft, KFO 257 grant TP1 and TP2, as well as by the SFB643 grants B9 and GRK1071. We thank S. Rössner, C. Kuhnt, S. Wallmüller, and K. Kuczera for technical assistance. Dr K. Prechtel performed the proofreading of the manuscript.

\section{DISCLOSURE}

The authors declared no conflict of interest.

c) 2014 Society for Mucosal Immunology

\section{REFERENCES}

1. Garrett, W.S., Gordon, J.I. \& Glimcher, L.H. Homeostasis and inflammation in the intestine. Cell 140, 859-870 (2010).

2. Strober, W., Fuss, I. \& Mannon, P. The fundamental basis of inflammatory bowel disease. J. Clin. Inv 117, 514-521 (2007).

3. Laroux, F.S. \& Grisham, M.B. Immunological basis of inflammatory bowel disease: role of microcirculation. Microcirculation 8, 283-301 (2001).

4. Sartor, R.B. Microbial influences in inflammatory bowel diseases. Gastroenterology 134, 577-594 (2008).

5. Colombel, J.F. et al. Infliximab, azathioprine, or combination therapy for Crohn's disease. N. Engl. J. Med. 5, 1383-1395 (2010).

6. Prechtel, A.T., Turza, N.M., Theodoridis, A.A. \& Steinkasserer, A. CD83 knockdown in monocyte-derived dendritic cells by small interfering
RNA (siRNA) leads to a diminished T-cell stimulation. J. Immunol 178, 5454-5464 (2007).

7. Prazma, C.M., Yazawa, N., Fujimoto, Y., Fujimoto, M. \& Tedder, T.F. CD83 expression is a sensitive marker of activation required for $\mathrm{B}$ cell and CD4 + T cell longevity in vivo. J. Immunol 179, 4550-4562 (2007).

8. Hock, B.D., Kato, M., McKenzie, J.L. \& Hart, D.N. A soluble form of CD83 is released from activated dendritic cells and B lymphocytes, and is detectable in normal human sera. Int. Immunol 13, 959-967 (2001).

9. Hock, B.D., Haring, L.F., Steinkasserer, A., Taylor, K.G., Patton, W.N. \& McKenzie, J.L. The soluble form of CD83 is present at elevated levels in a number of hematological malignancies. Leuk. Res. 28, 237-241 (2004).

10. Hock, B.D., Fernyhough, L.J., Gough, S.M., Steinkasserer, A., Cox, A.G. \& McKenzie, J.L. Release and clinical significance of soluble CD83 in chronic lymphocytic leukemia. Leuk. Res. 33, 1089-1095 (2009).

11. Sénéchal, B., Boruchov, A.M., Reagan, J.L., Hart, N. \& Young, J.W. Infection of mature monocyte derived dendritic cells with human cytomegalovirus inhibits stimulation of T-cell proliferation via the release of soluble CD83. Blood 103, 4207-4215 (2004).

12. Lechmann, M. et al. The extracellular domain of CD83 inhibits DCmediated T cell stimulation and binds to a ligand on dendritic cells. J. Exp. Med 194, 1813-1821 (2001).

13. Scholler, N., Hayden-Ledbetter, M., Dahlin, A., Hellström, I., Hellström, K.E. \& Ledbetter, J.A. CD83 regulates the development of cellular immunity. J. Immunol. 168, 2599-2602 (2002).

14. Lan, Z. et al. Induction of kidney allograft tolerance by soluble CD83 associated with prevalence of tolerogenic dendritic cells and indoleamine 2, 3-dioxygenase. Transplantation 90, 1286-1293 (2010).

15. Zinser, E., Lechmann, M., Golka, A., Lutz, M.B. \& Steinkasserer, A. Prevention and treatment of experimental autoimmune encephalomyelitis (EAE) by soluble CD83. J. Exp. Med. 200, 345-351 (2004).

16. Xu, J.F. etal. A limited course of soluble CD83 delays acute cellular rejection of MHC-mismatched mouse skin allografts. Transpl. Int 20, 266-276 (2007).

17. Ge, W. et al. Immunosuppression involving soluble CD83 induces tolerogenic dendritic cells that prevent cardiac allograft rejection. Transplantation 90, 1145-1156 (2010).

18. Lechmann, M., Shuman, N., Wakeham, A. \& Mak, T.W. The CD83 reporter mouse elucidates the activity of the CD83 promoter in $\mathrm{B}, \mathrm{T}$, and dendritic cell populations in vivo. Proc. Natl. Acad. Sci. USA 105, 11887-11892 (2008).

19. Hawkins, J.V. et al. Protease activity in a hapten-induced model of ulcerative colitis. Dig. Dis. Sci 42, 1969-1980 (1997).

20. Neurath, M.F., Fuss, I., Kelsall, B.L., Stüber, E. \& Strober, W. Antibodies to interleukin 12 abrogate established experimental colitis in mice. J. Exp. Med. 182, 1281-1290 (1995).

21. Spadoni, I., lliev, I.D., Rossi, G. \& Rescigno, M. Dendritic cells produce TSLP that limits the differentiation of Th17 cells, fosters Treg development, and protects against colitis. Mucosal Immunol 5, 184-193 (2012).

22. Reardon, C. et al. Thymic stromal lymphopoetin-induced expression of the endogenous inhibitory enzyme SLPI mediates recovery from colonic inflammation. Immunity 35, 223-235 (2011).

23. Munn, D.H., Shafizadeh, E., Attwood, J.T., Bondarev, I., Pashine, A. \& Mellor, A.L. Inhibition of T cell proliferation by macrophage tryptophan catabolism. J. Exp. Med. 189, 1363-1372 (1999).

24. Mellor, A.L. \& Munn, D.H. IDO expression by dendritic cells: tolerance and tryptophan catabolism. Nat. Rev. Immunol. 4, 762-774 (2004).

25. Pallotta, M.T. et al. Indoleamin 2,3-dioxygenase is a signaling protein in long-term tolerance by dendritic cells. Nat. Immunol 12, 870-878 (2011).

26. Okamoto, T., Toné, S., Kanouchi, H., Miyawaki, C., Ono, S. \& Minatogawa, Y. Transcriptional regulation of indoleamine 2, 3-dioxygenase (IDO) by tryptophan and its analogue. Cytotech 54, 107-113 (2007).

27. Pithadia, A.B. \& Jain, S. Treatment of inflammatory bowel disease (IBD). Pharmacol. Reports 63, 629-642 (2011).

28. Gonzalez-Rey, E., Chorny, A. \& Delgado, M. Regulation of immune tolerance by anti-inflammatory neuropeptides. Nat. Rev. Immunol. 7, 52-63 (2007)

29. Abad, C. et al. cDNA array analysis of cytokines, chemokines, and receptors involved in the development of TNBS-induced colitis: homeostatic role of VIP. Inflamm. Bowel. Dis 11, 674-684 (2005). 


\section{ARTICLES}

30. Arranz, A., Abad, C., Juarranz, Y., Leceta, J., Martinez, C. \& Gomariz, R.P. Vasoactive intestinal peptide as a healing mediator in Crohn's disease. Neuroimmunomodulation 15, 46-53 (2008).

31. Chen, W.J. IDO: more than an enzyme. Nat. Immunol. 12, 809-811 (2011).

32. Breloer, M. \& Fleischer, B. CD83 regulates lymphocyte maturation, activation and homeostasis. Trends Immunol. 29, 186-194 (2008).

33. Wirtz, S. \& Neurath, M.F. Mouse models of inflammatory bowel disease. Adv. Drug Deliv. Rev. 59, 1073-1083 (2007).

34. Massa, F. et al. The endogenous cannabinoid system protects against colonic inflammation. J. Clin. Invest 113, 1202-1209 (2004).

35. Reardon, C., Wang, A. \& McKay, D.M. Transient local depletion of Foxp3+ regulatory $T$ cells during recovery from colitis via Fas/Fas ligand-induced death. J. Immunol 180, 8316-8326 (2008).

36. Bouma, G. \& Strober, W. The immunological and genetic basis of inflammatory bowel disease. Nat. Rev. Immunol. 3, 521-533 (2003).

37. Taylor, M.W. \& Feng, G.S. Relationship between interferon-gamma, indoleamine 2, 3-dioxygenase, and tryptophan catabolism. FASEB J 5 2516-2522 (1991).

38. Bock, F. et al. Topical application of soluble CD83 induces IDO-mediated immune modulation, increases Foxp3 + T cells, and prolongs allogeneic corneal graft survival. J. Immunol. 191, 1965-1975 (2013).
39. Gurtner, G.J., Newberry, R.D., Schloemann, S.R., McDonald, K.G. \& Stenson, W.F. Inhibition of indoleamine 2,3-dioxygenase augments trinitrobenzene sulfonic acid colitis in mice. Gastroenterology 125, 1762-1773 (2003).

40. Van der Sluijs, K., Singh, R., Dijkhuis, A., Snoek, M. \& Lutter, R. Indoleamine-2,3-dioxygenase activity induces neutrophil apoptosis. Crit. Care 15 (Suppl 1), P208 (2011).

41. Hunter, M.M., Wang, A., Hirota, C.L. \& McKay, D.M. Neutralizing anti-IL-10 antibody blocks the protective effect of tapeworm infection in a murine model of chemically-induced colitis. J. Immunol 174, 7368-7375 (2005).

42. Xu, Y. et al. Bioprocess development for production, purification, and structural characterization of recombinant hCD83ext as a potential therapeutic protein. Protein Expr. Purif 65, 92-99 (2009).

43. Becker, C., Fantini, M.C. \& Neurath, M.F. High resolution colonoscopy in live mice. Nat. Prot 1, 2900-2904 (2006).

44. Schubert, W. et al. Analyzing proteome topology and function by automated multi-dimensional fluorescence microscopy. Nat. Biotechnol 24, 1270-1278 (2006).

45. Eckhardt, J., Ostalecki, C., Kuczera, K., Schuler, G., Pommer, A.J. \& Lechmann, M. Murine whole-organ immune cell populations revealed by multiepitope-ligand cartography. J. Histochem. Cytochem. 61, 125-133 (2013). 